\title{
Shear Stress Measurements of Non-Spherical Particles in High Shear Rate Flows
}

\author{
Erin Koos, Melany L. Hunt and Christopher E. Brennen \\ California Institute of Technology; MC 104-44; Pasadena, California 91125, USA
}

\begin{abstract}
The behavior of liquid-solid flows varies greatly depending on fluid viscosity; particle and liquid inertia; and collisions and near-collisions between particles. Shear stress measurements were made in a coaxial rheometer with a height to gap ratio $\left(b / r_{o}\right)$ of 11.7 and gap to outer radius ratio $(h / b)$ of 0.166 that was specially designed to minimize the effects of secondary flows. Experiments were performed for a range of Reynolds numbers, solid fractions and ratio of particle to fluid densities. With neutrally buoyant particles, the dimensional shear stress exhibits a linear dependence on Reynolds number: the slope is monotonic but a non-linear function of the solid fraction. Though non-neutrally buoyant particles exhibit a similar linear dependence at higher Reynolds numbers, at lower values the shear stress exhibits a non-linear behavior in which the stress increases with decreasing Reynolds number due to particle settling.
\end{abstract}

Keywords: Rheology, Suspension

PACS: $83.80 . \mathrm{Hj}$

\section{INTRODUCTION AND EXPERIMENTAL PROCEEDURE}

Fluid-solid flows are observed in a variety of fields ranging from mining operation to the erosion of the Martian landscape. These flows were first studied by Bagnold who found that there was a transition from the linear "macroviscous region" to the quadratic "grain-inertia region" [1,2]. Later analysis by Hunt et. al found that Bagnold's experiments were marred by boundary layer growth from the the annular end-caps and secondary vortices at high Reynolds numbers (in the "grain-inertia region") [3]. Work by Chen and Ling, found that the higher volume fractions tested by Bagnold ( $\phi=0.606,0.623$ ), were inconsistent due to particle slip against the cylinder walls [4]. The present experiment attempts to avoid these difficulties. The flows are primarily governed by four non-dimensional parameters: the solid fraction of particles, the Reynolds number, the Péclet number and the Bagnold number. If, as in the following experiments, both the Peclet and Reynolds numbers can be neglected, the flow will be Newtonian and only depend on the volume fraction of particles.

The coaxial shear cell, Figure 1a), consists of a fluid-particle mixture confined between two stainless steel concentric

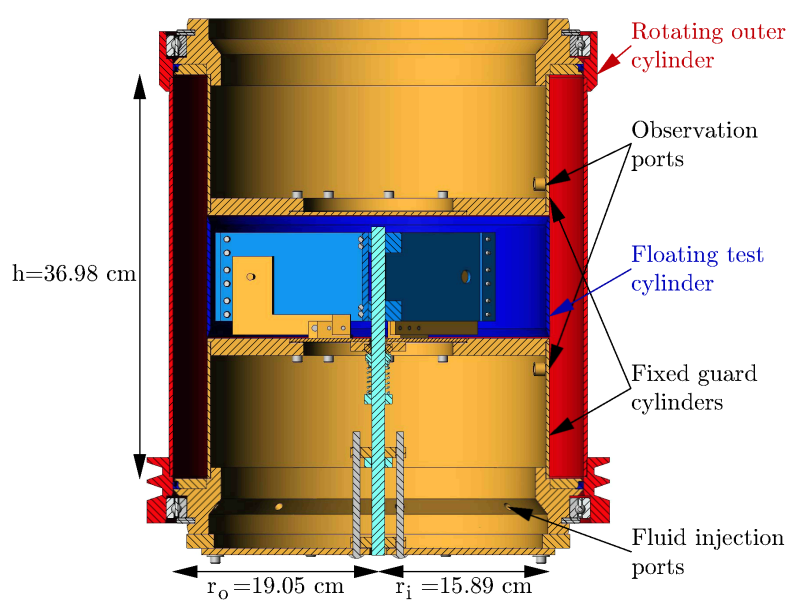

(a)

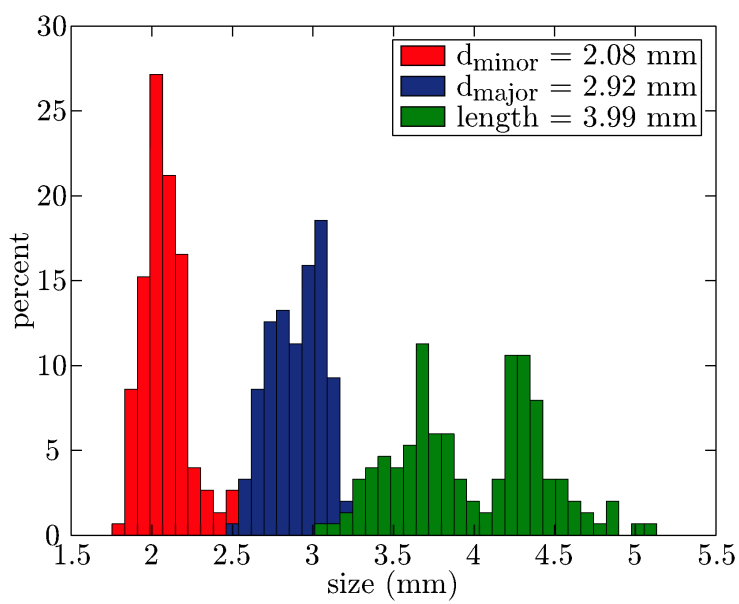

(b)

FIGURE 1. (a) Coaxial rotating cylinder, Couette flow device. The outer cylinder (red) rotates while the inner cylinder remains fixed. (b) Histogram of polystyrene particle sizes showing the major and minor diameters of the ellipse and the cylinder length. 
cylinders. The inner cylinder consists of three sections: two fixed guard sections; and a floating section that deflects rotationally for torque measurements. This annular Couette flow device was designed to reduce the effect of secondary vortices on fluid measurements: the flow is driven by outer cylinder rotation, the radii are large in comparison to the gap, and measurements are made in the center so as to reduce any effects from the endcaps. Two observation ports, located on the inner fixed cylinders $2.86 \mathrm{~cm}$ above and below the floating test cylinder, are used with optical probes to measure the effective volume fraction in the annular gap. By normalizing the measured steady-state torque by the pure fluid torque, the effective viscosity ratio $\mu^{\prime} / \mu$ is found. For Newtonian fluids, both the shear and torque vary linearly with the Reynolds number $\left(\mathrm{Re}=\rho \dot{\gamma} b^{2} / \mu\right)$, and the effective viscosity ratio is a constant.

Polystyrene is a colorless hard plastic used, for example, in injection molding processes with a density of 1050 $\mathrm{kg} / \mathrm{m}^{3}$ (a matched density aqueous glycerine mixture has $21 \%$ glycerine). The polystyrene particles are elliptical cylinders with unimodal radii and bimodal lengths as seen in Figure $1 \mathrm{~b}$. The polystyrene particles have a spherical equivalent radius of $r_{p}=1.67 \pm 0.01 \mathrm{~mm}$. Random close packing, $\phi_{m}$ for the polystyrene particles was measured using the displaced volume of a sample of polystyrene as $65 \%$ of the total volume.

\section{EXPERIMENTS}

We first describe the experimental data for polystyrene particles in a matched density fluid of $21 \%$ glycerine, where the volume fraction of particles was varied while the resulting shear stress $\tau$ on the inner, floating cylinder was calculated from measurements of the torque and shown in Figure 2a. Each point represents the mean value of at least five individually recorded measurements shown with error bars representing the standard deviation in these measurements. For each volume fraction, the shear stress is shown with its linear fit. The shear stress increases rapidly with with volume fraction: varying by several orders of magnitude between the smallest and largest volume fraction where the dependance on the volume fraction appears to be more pronounced as the volume fraction increases.

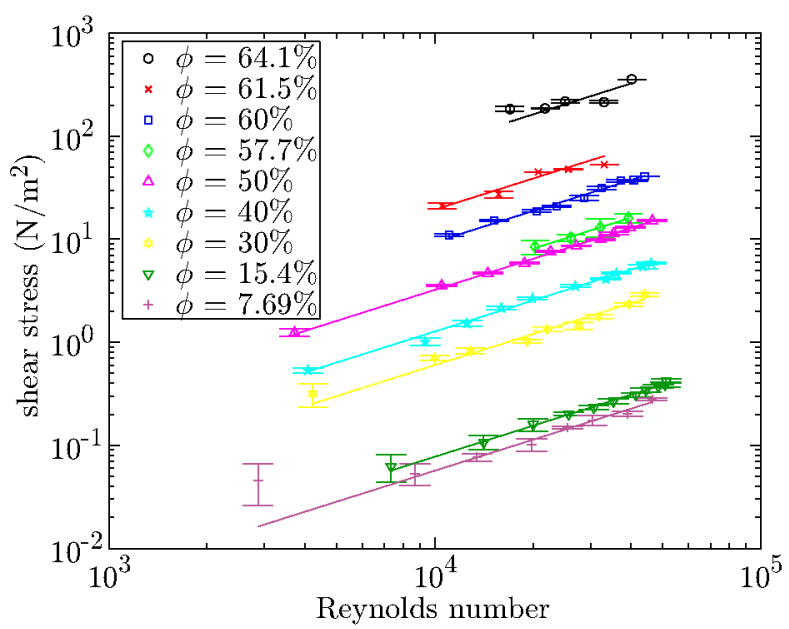

(a)

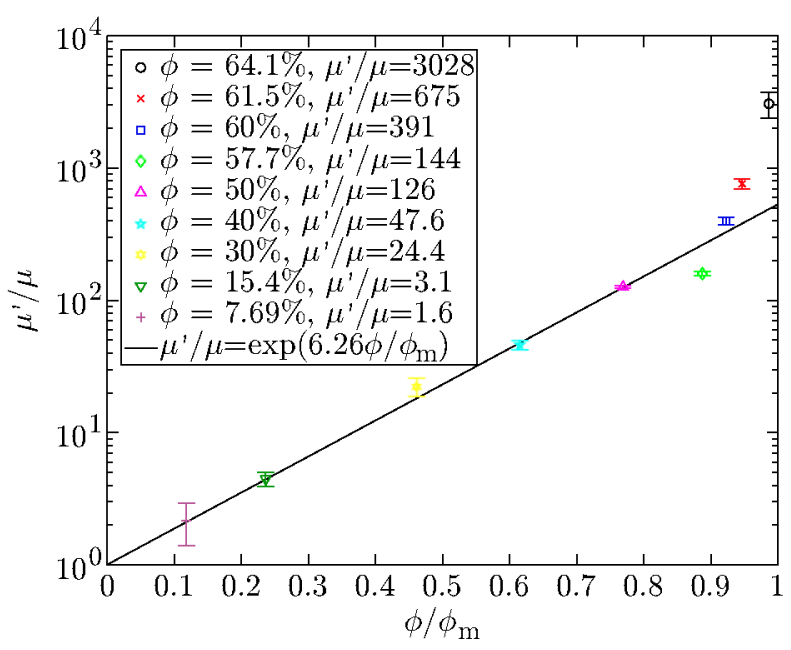

(b)

FIGURE 2. (a) Shear stress measurements for suspensions of polystyrene particles in aqueous glycerine. The lines represent linear fits for each volume fraction, fits that are constrained to pass through the origin. (b) Effective viscosity ratio for neutrally buoyant polystyrene particles in aqueous glycerine solutions. The black line is an exponential fit for the points below $\phi=0.60$

The effective viscosity is a function of the volume fraction, but does not appear to vary greatly on the Reynolds number, as expected with a Newtonian fluid. For the two lowest volume fractions $(\phi=0.077$ and $\phi=0.154 \%)$, the effective viscosity does increase slightly with Reynolds number, which may be due to the onset of secondary flows in the fluid. The effective viscosity ratio is shown as a function of the volume fraction in Figure $2 b$, where the volume fraction of solids is normalized against the maximum volume fraction $\left(\phi_{m}=0.65\right)$. For points below $\phi=0.60$, the effective viscosity is fitted well by an exponential. For larger volume fractions, the effective viscosity grows more quickly than the exponential. This deviation is expected as the particles near a region of jamming.

In the second set of experiments with non-neutrally buoyant particles in a Newtonian fluid, the volume fraction of solids has a dramatic influence on the effective viscosity of the fluid-particulate mixture. For particles with a density different than the interstitial fluid, the particles will tend to move away from the floating cylinder (where the shear stress 
measurements are taken). As the particles migrate away from the central, floating cylinder, the local volume fraction decreases, a change that should reduce the effective viscosity ratio. As with the previous experiments, this effective viscosity does not depend on the Reynolds number, but it does depend on the fluid density as seen in Figure $3 a$. For each volume fraction, the effective viscosity ratio is highest when the fluid and particle densities match. As was

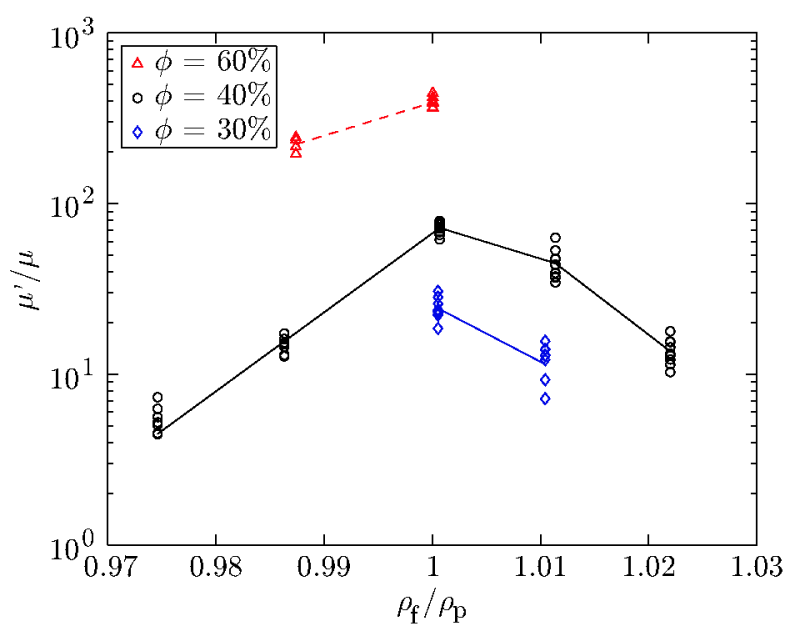

(a)

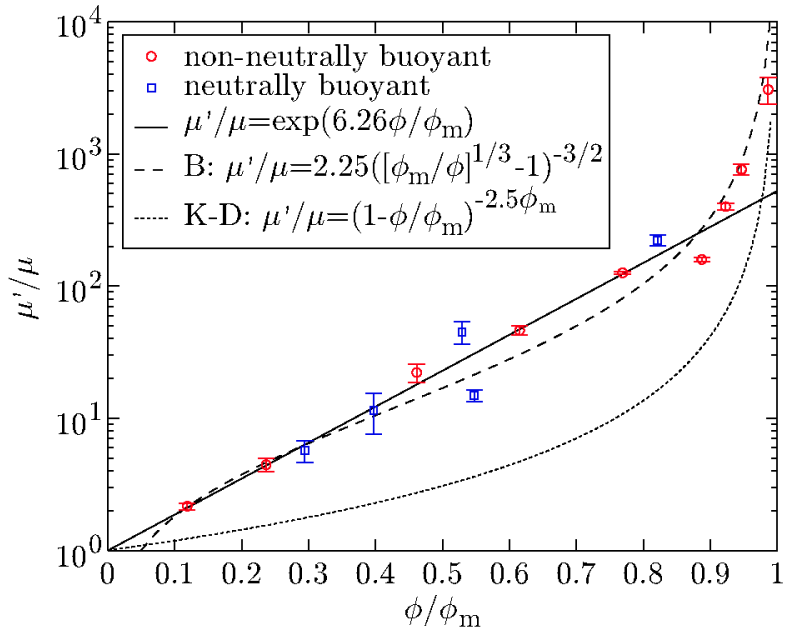

(b)

FIGURE 3. (a) Effective viscosity ratio as a function of the density of the interstitial fluid normalized by the particle density. (b) Effective viscosity ratio for neutrally buoyant and non-neutrally buoyant experiments of polystyrene particles in aqueous glycerine solutions shown with relations proposed by B: Bagnold and K-D: Krieger and Dougherty.

hypothesized previously, as the density ratio diverges from unity, the particles will float or sink away from the central cylinder. As the volume fraction decreases around this center section, the measured torque will also decrease. As the interstitial fluid is varied, the particle velocity and particle count per second are measured using the optical sensors. The local volume fraction is found by assuming a distribution of particles that varies only axially within the annular gap. By adjusting for the actual volume fraction across the floating cylinder, the effective viscosity for non-neutrally buoyant particles should match the results obtained for neutrally buoyant particles as seen in Figure $3 \mathrm{~b}$. This data is also compared to the linear relation proposed by Bagnold [2] and the model of Krieger and Dougherty [5].

\section{CONCLUSIONS}

The effective viscosity for mixtures of neutrally buoyant and non-neutrally buoyant polystyrene particles in aqueous glycerine is a function the local volume fraction of solids only. For the range of Reynolds numbers and volume fractions studied, these steady-state flows are Newtonian. The effective viscosity grows exponentially for low volume fractions $\left(\phi / \phi_{m}<0.8\right)$ and greater than exponentially for higher volume fractions and compares well with the linear macro-viscous relation proposed by Bagnold.

\section{ACKNOWLEDGMENTS}

This research was supported though the generous support of NASA (NNC04GA48G) and the NSF (CBET-0730284).

\section{REFERENCES}

1. R. Bagnold, Proc. R. Soc. Lond. Ser. A 225, 49-53 (1954).

2. R. Bagnold, Philos. Trans. R. Soc. Lond. Ser. A 249, 235-297 (1956).

3. M. Hunt, R. Zenit, C. Campbell, and C. Brennen, J. Fluid Mech. 452, 1-24 (2002).

4. C. Chen, and C. Ling, Journal of Engineering Mechanics, ASCE 122, 469-480 (1996).

5. I. Krieger, and T. Dougherty, Transactions of the Socieity of Rheology 3, 137-152 (1959). 